AVANT-PROPOS

\title{
POUR UNE HISTOIRE DU PRINCIPE DE CIRCULATION EN EUROPE
}

\author{
Henriette AssÉO
}

\begin{abstract}
«Ce qui pourra sembler un peu bizarre à l'avenir, c'est que nos dissidences en 1848, les plus âpres peut-être, étaient relatives au passé, historiques, archéologiques. »
\end{abstract}

Jules MiCHELET $^{1}$

Le développement d'une mentalité obsidionale devant les dangers de la mondialisation ébranle une confiance déjà largement érodée dans l'universalisme émancipateur de l'Occident. Toute une littérature de la déploration succède, avec la même prétention à dire le vrai, à l'ancienne glorification de notre continent. Et l'européanisme sera volontiers confondu avec l'atlantisme, instrument pervers de la pénétration américaine. Ces craintes révèlent les difficultés éprouvées par les Européens à concevoir la mobilité maîtrisée des hommes comme une ambition de civilisation commune. C'est pourtant la remise en mouvement des sociétés européennes qui a entraîné le démantèlement définitif du « rideau de fer » quand, le 9 novembre 1989, le porte-parole du gouvernement d'Egon Krenz, Günther Schabowski, prit l'initiative d'annoncer à la toute dernière minute d'une conférence de presse retransmise en direct à la télévision, un « décret sur les voyages », qui prenait effet immédiatement. Cette nuit de novembre ouvrait une ère nouvelle, matérialisée par la chute du mur de Berlin et onze mois plus tard, au terme d'intenses négociations, l'Allemagne était réunifiée. Pour la première fois depuis des siècles, l'Europe connaissait un bouleversement pacifique de son équilibre géopolitique.

1. Jules Michelet, Histoire de la Révolution française, t. I, préf. de 1868, Paris, J. Hetzel et $\mathrm{C}^{\mathrm{ie}}$, s. d., p. III. 
Pourtant l'obligation qui nous est faite de penser l'Europe comme un territoire en formation ne va pas de soi pour l'historien. Il perçoit bien l'importance capitale des événements actuels sans pour autant chercher à organiser leur principe d'appartenance selon de nouvelles grilles d'analyse. Il lui suffira de parler de la fin des grands héritages des $\mathrm{XIX}^{\mathrm{e}}$ et $\mathrm{XX}^{\mathrm{e}}$ siècles, qu'il considère les rapports entre nations et nationalités, entre économie dirigée et mondialisation, entre information cantonnée et globalisée, entre blocs militaires et équilibre de la puissance, entre guerres et pacification. Tous ces faits peuvent s'inscrire dans une perspective de plus ou moins longue durée, certes éclairante ; mais éclairante de quoi ? Car nous gardons une fâcheuse tendance à interroger le passé selon des angles de vue prédéterminés par une immersion professionnelle constitutive des habitudes de pensée, en particulier la crainte partagée par les meilleurs esprits de blesser la mémoire nationale, dont, en Europe, les historiens sont naturellement les gardiens vigilants ${ }^{2}$.

Quelques exemples, empruntés à l'histoire européenne récente, permettront de montrer que le statut de fait historique en formation est difficile à identifier.

Quels liens complexes existe-t-il en effet entre le « décret sur les voyages », le retour, militairement exigé, des Kosovars dans leur pays, les raidissements nationaux de l'Autriche ou le vote du 21 avril 2002 en France ? Si ce n'est de constater qu'aucun peuple n'est déterminé dans ses choix par un horizon insurmontable de tempérament politique ou culturel.

Reprenons les exemples cités plus haut. Le politologue qui veut analyser la portée du « décret sur les voyages » jugerait stupide d'en limiter l'examen à la seule sphère de la circulation des hommes et de l'usage politique du tourisme. Cette décision s'intègre par son opportunisme politique dépassant d'ailleurs la capacité d'analyse des acteurs de l'époque dans un processus historique de grande ampleur, celui de la chute du mur de Berlin et de la réunification allemande, devenu, curieusement, aussi évident à la conscience historienne qu'il fut ignoré par elle pendant des décennies. Si les enjeux du voyage ont largement été étudiés pour les siècles passés, si l'on accorde au Grand Tour le label politique des Lumières, pourquoi ne pas situer les poussées motrices intra-européennes à leur véritable niveau d'interprétation ? On pourrait ainsi observer un renversement structurel des mobilités collectives européennes plus ou moins forcées, y compris par la descente aux abysses de l'extermination, vers une mobilité intraeuropéenne individuelle ou familiale, voulue - ce qui ne veut pas dire heureuse -,

2. Marcel Detienne, Comparer l'incomparable, Paris, Seuil (La Librairie du $\mathrm{XX}^{\mathrm{e}}$ siècle), 2000, p. 58-59. 
qui recommande à long terme la réorganisation sociale du système territorial européen ${ }^{3}$.

Décret sur les voyages versus droit d'asile. Laisser partir ou faire revenir? La gestion internationale du premier conflit militaire de l'après-guerre froide celui de la Yougoslavie -, et de la question des réfugiés du Kosovo, appelle des remarques d'importance sur l'imaginaire de la mobilité et son refoulement. La politique de l'espace européen me semble guidée par des principes contradictoires venus des profondeurs de l'inconscient politique de la mentalité occidentale. La coexistence de communautés, voire de ghettos, est tolérée à condition d'éviter la formation durable de campements de masse de réfugiés. Ce n'est certainement pas Auschwitz, l'horizon insurmontable des Européens, mais les flux gigantesques de populations induites par les deux guerres mondiales. Sur le territoire européen, il ne peut y avoir de camps de réfugiés permanents, il convient de laisser la place au départ et au retour. Ainsi, la délimitation matérielle du futur empire européen ne présente ni centre ni périphérie ; ses frontières mouvantes dessinent des méandres qui englobent une zone d'attente dans un aéroport, ou les confins embrasés des frontières militaires impériales de la Russie ou de la Turquie. Le droit d'asile se transforme avec cynisme en une gestion communautaire du retour vers les « pays sûrs », si possible non européens.

Les aléas récents du suffrage démocratique sont-ils aussi révélateurs des enjeux nouveaux de la mobilité ? Si l'Autriche trouve les souvenirs du paritarisme encombrant, la Croatie, fauteuse de guerre il n'y a pas si longtemps, espère renouer par l'ouverture démocratique avec un très ancien dispositif de stabilisation des Balkans. Dans ces deux cas, l'héritage de l'Empire austrohongrois est reformulé de façon complexe pour laisser à ces pays réduits aux dimensions médiocres de régions centrales une marge de manœuvre leur permettant de peser sur l'Europe en mouvement. La digestion des idées de l'extrême droite et de son « hôte muet », Jörg Haider, par les Autrichiens peut bien se lire comme le retour d'un «nationalisme de rétraction », fixé sur les questions sensibles de la citoyenneté et de l'immigration. Sauf à considérer que toutes les nations d'Europe seront confrontées à la requalification de leur citoyenneté pour reconstituer à un niveau de territoire différent la fiction d'un peuple souverain ! Les choix politiques de l'arc alpin ont donné le signal de la réouverture de la question communautaire comme une question nationale et

3. Il importe de suivre, à la fois, les propositions de Klaus BADE : « L'histoire des migrations doit donc toujours aussi être comprise comme une partie de l'histoire générale, et uniquement dans ce contexte. Cela vaut aussi pour l'histoire européenne des migrations », in ID., L'Europe en mouvement. La migration de la fin du XVIII siècle à nos jours, trad. de l'allemand par Olivier Mannoni, Paris, Seuil, 2002, p. 8, et celles de Nancy GreEn sur le multicomparatisme exposées in ID., Repenser les migrations, Paris, Presses universitaires de France (Le Nœud gordien), 2002, chap. II, p. 23-35, tout particulièrement « Pour un multiculturalisme », p. 33-35, pour revoir le dossier des relations entre la formation des États et la mobilité en Europe. 
démocratique en Europe occidentale, en parfaite symétrie avec les enjeux politiques de l'Europe orientale. La crise du système constitutionnel français, un révisionnisme timoré qui a succédé au temps long de la sacralisation, s'intègre parfaitement dans cette problématique. Les Français ont donné un avertissement à leurs gouvernants en relisant le programme de Jean-Marie Le Pen à la lumière de la démocratie. Ils ont bien compris que la constitution européenne ne pourrait pas s'exercer dans le cadre institutionnel existant, et ils ont eu, sans doute, peur du vide. Il eût convenu d'aborder avec imagination cette question fondamentale, mais le suffrage universel n'a pas donné de réponse à cette question parce que les Politiques ne l'ont pas posée.

Ces formes de délitement du pacte républicain de pacification seraient le symptôme d'une implosion éventuelle des systèmes démocratiques à base nationale. Mais la pensée intellectuelle dite « critique » garde la fâcheuse habitude d'accompagner les mouvements de fond en croyant les dénoncer. Ainsi, dans leur volonté de dépassement des souverainetés nationales, devenues à leurs yeux obsolètes, les contempteurs du repli sur soi, apôtres des nouveaux internationalismes, franchissent le pas de l'Europe qu'ils s'acharnent à faire confondre par les âmes simples avec l'Occident colonialiste, pour élaborer un nouveau droit international mondial qui s'imposerait à tous par le biais d'instances morales.

«Les usages politiques du passé » se révèlent aussi complexes à manier en ces temps démocratiques et ouverts qu'au temps des dictatures et des totalitarismes ${ }^{4}$. Changer de siècle et changer de système de souveraineté par des voies relativement pacifiques et ouvertes sont des opérations inédites de concaténation du temps et de l'espace qui reposent sur des processus dont la finalité n'est pas maîtrisée et dont la généalogie demeure obscure. On peut en appeler à l'universalisme chrétien, ou encore, ce qui reste d'assez mauvais goût, évoquer l'idéal paneuropéen d'un conquérant (même éclairé). Le jugement historique, déjà aux prises avec la rude épreuve de la révision des récits nationaux, la digestion d'un passé qui ne passe pas, la nostalgie d'illusions tenaces, découvre pour la première fois son inaptitude à lire l'avenir à la lumière du passé.

Dans ces choix aventurés, on tend à oublier les raisons qui ont présidé à la sacralisation de la nation. On voit bien l'impossibilité de transférer l'inflexible généalogie proposée par Jules Michelet et Ernest Lavisse aux dimensions du nouvel empire européen par une simple méthode extensive, tandis que se développent les histoires particulières qui n'éprouvent nullement le besoin d'être compatibles entre elles. Le noble rêve de l'objectivité et de « la restauration de la continuité au présent » avait contraint la génération des historiens français de 1820 à inventer les moyens de rétablir une passerelle par-dessus la cassure

4. Il convient de lire de près l'ensemble des réflexions autour de ce thème, in Jacques Revel et François Hartog, Les Usages politiques du passé, Paris, Éditions de l'École des hautes études en sciences sociales (Enquête), 2001. 
radicale que la Révolution avait inscrite dans le destin national. Mais la conciliation entre l'histoire romantique et l'histoire critique n'a jamais eu lieu et Charles Péguy, dans sa sévérité de jugement à l'égard d'Ernest Lavisse, pouvait, à bon droit, rappeler qu'il n'existe nulle « tranquillité » pour l'historien dont le rôle est moins de prononcer des jugements historiques que d'en élaborer constamment de nouveaux ${ }^{5}$.

Le projet de Lucien Febvre pour l'après-guerre, exposé dans son cours au Collège de France pendant l'année 1944-1945, n'a pas été réalisé6 ${ }^{\text {. L'Europe }}$ ne devait pas être, pour lui, un concept géographique, mais une communauté de civilisation. Par contre, le fractionnement des thèmes en enjeux structuraux, chargés de pesanteurs, a donné aux historiens de l'école française une énergie créatrice extraordinaire dans l'entreprise de reformulation de l'histoire nationale en une « geste » intégrale qui n'a pas eu son équivalent.

Mais de quels héritages informulés sommes-nous restés inconsciemment tributaires? La difficulté éprouvée par les historiens pour réfuter la rhétorique péremptoire du relativisme postmoderne viendrait de la méfiance à l'égard de toute approche de l'histoire européenne par la morphologie culturelle. Faut-il renoncer à l'effort de mise à distance de tous les événements récents dans un passé que le récit construirait par le jeu rhétorique de la concordance des temps comme irrémédiablement révolu ? C'est le régime d'historicité « présentiste », observé par François Hartog, qui favorise l'idée que la stagnation mémorielle est un « ordre raisonné du passé ». Si les rapports entre l'histoire et la mémoire ont été discutés avec intensité, « il reste cependant que l'historiographie et la mémoire ne convergent pas nécessairement », affirme Carlo Ginzburg, avant d'ajouter : « [...] et je voudrais justement ici souligner un aspect différent du problème, que l'on pourrait même dire à contre-courant du discours ordinaire : l'irréductibilité de la mémoire à l'histoire ${ }^{7}$. »

S'il existe de multiples usages de la référence à l'Europe, nul ne se risque à proposer un patriotisme européen dynamique ${ }^{8}$. Alors, nous rêvons, sans trop y

5. Voir la nouvelle préface de François HARTOG à son livre, Le XIXe Siècle et l'histoire. Le cas Fustel de Coulanges, Paris, Seuil, 2001.

6. L'Europe, genèse d'une civilisation, cours professé par Lucien FEBVRE au Collège de France en 1944-1945, établi, présenté et annoté par Thérèse CHARMASSON et Brigitte MAZON, avec la collab. de Sarah LüDEMAn, préf. de Marc FerRo, Paris, Perrin, 1999.

7. Carlo GinZBURG, À distance. Neuf essais sur le point de vue en histoire, trad. de l'italien par Pierre-Antoine FABRE, Paris, Gallimard (Bibliothèque des histoires), 2001, p. 148.

8. La remarque, formulée en 1990 par Anne-Sophie PERriaUX, est toujours vérifiée : « À titre d'hypothèse, il faut imaginer que l'une des variations du thème ("la culture européenne") s'est moins substituée aux "cultures nationales", qu'elle ne leur a permis de subsister », in ID., «La Communauté économique européenne, les États et la culture, 1957-1987 », Revue de synthèse, $\mathrm{n}^{\circ}$ spéc. " La difficile institution de l'Europe », 4e sér., 3, juil.-sept. 1990, p. 271-287, en part. p. 287. 
croire, au cosmopolitisme des Lumières comme dispositif fondateur d'une manière sensible de tenir l'espace de civilité par la proximité des mœurs, en oubliant que le parcours matériel (réalisé et/ou rêvé par le récit des Lumières et les sociabilités du Grand Tour) permettait à une société tout entière, comme le montre, dans cette livraison, Daniel Roche, et pas uniquement à ses élites, de procéder à cette intériorisation, prélude aux grandes espérances. Jean-Louis Carra donnait à voir le triptyque des représentations du monde policé : «L'Europe est aujourd'hui devenue le théâtre de grands événements, et pour ainsi dire, le siège de l'Empire du monde »; et il poursuit l'apologétique du territoire des Lumières :

«La France, l'Angleterre, une partie de l'Allemagne et de l'Italie occupent le centre de ce continent, et de ce centre partent les lumières qui vont éclairer les autres contrées du globe [...] nul ne s'est fait une gloire de parcourir, en observateur philosophique et politique tout ensemble, l'Allemagne, l'Angleterre, l'Italie, la Suède, le Danemark, la Hongrie, la Transilvanie [sic], la Pologne, l'Ukraine, la Grande Russie et les provinces européennes de l'Empire ottoman ${ }^{9}$. »

La circulation des hommes comme celle des biens était alors envisagée comme un échange, la main invisible de l'échange général, plus que comme un mouvement brownien; le commerce doux entre les nations devait assurer la paix du monde. Mais Pierre-Yves Beaurepaire nous rappelle ici que des loges maçonniques, qui avaient pour ambition de décliner cette utopie de fraternité planétaire, ont renoncé pourtant, dès la fin du XVIII ${ }^{\mathrm{e}}$ siècle, à un « ordre cosmopolite et neutre » pour céder aux tentations nationalistes.

La pensée romantique a voulu reformuler le déplacement, et ancrer le marcheur impénitent dans la géologie pour lui laisser la tête à la généalogie et à la quête des origines, et le libérer ainsi des prétentions artificielles de la sociabilité aristocratique. Mais il s'est alors développé l'idée que le déracinement impliquait le déclassement. Le cosmopolitisme, tout comme le génie indompté du Geniezeit du Sturm und Drang, est devenu un « gêneur public ». La stigmatisation des identités cosmopolites a succédé à la valorisation des pratiques cosmopolites. Les ordres nationaux sont par nature instables ; ils ont favorisé le développement des fausses figures du «nous » dont parle Nietzsche ${ }^{10}$. Les groupes minoritaires ont adopté des conduites collectives fixistes qui en rajoutaient dans l'universalisme sans assignation ou dans la crispation particulariste. Sans doute le refoulement du

9. Histoire de la Moldavie et de la Valachie avec une dissertation sur l'état actuel de ces deux provinces. Par M. C... [Jean-Louis Carra] qui a séjourné dans ces provinces, à Jassy, aux dépens de la Société typographique des Deux Ponts, MDCCLXXVII, « discours préliminaire », p. V-XXX.

10. Marc CRÉPOn, Le Malin génie des langues (Nietzsche, Heidegger, Rosenzweig), Paris, Vrin (Problèmes et controverses), 2000, p. 94-95 : «Le repli des nations sur elles-mêmes n'est alors rien d'autre qu'une réaction névrotique au nivellement démocratique - une réaction chargée de ressentiment à ce que Nietzsche appelle par ailleurs, le "funeste destin de l'Europe". » 
cosmopolitisme empêchait de pouvoir penser autrement qu'en termes politiques les enjeux des déplacements de l'entre-deux-guerres. Arendt a théorisé l'anticipation intellectuelle qui fit croire aux Juifs allemands de l'entre-deuxguerres qu'une identité européenne de substitution pourrait remplacer la nationalisation impossible. Comme le montre ici Enzo Traverso, le cosmopolitisme judéo-allemand a pris les sombres couleurs de l'exil avec, pour tout bagage, les vestiges d'une Aufklärung inopérante. Pourtant, Pierre Hassner affirme, dans son article, et dans un entretien postérieur au 11 septembre 2001, la vigueur d'une pensée politique du cosmopolitisme conciliant Kant et Arendt. Il faudrait, comme Goethe, rester « un cosmopolite impénitent ${ }^{11}$ ».

Le cosmopolitisme des Lumières n'a pas eu de postérité européenne affichée. Il a laissé la place à un double mouvement d'occultation politique et intellectuelle du principe de circulation, par le jeu de l'interception à la frontière et par la manipulation du principe des nationalités. Ces procédures de refoulement ne disent rien de l'ampleur et de la variété des déplacements réels à travers l'Europe, mais elles ont focalisé sur les minorités migrantes la vision d'agents potentiels d'un démantèlement des ordres nationaux. Les Juifs européens ont payé un tribut considérable. Née à Salonique sous l'Empire ottoman, ma grand-mère paternelle s'appelait Henriette et elle s'exprimait en français comme toute ma famille. Elle était bien plus jeune que moi, qui écris ces lignes, quand elle est décédée dans le convoi qui la conduisait vers les territoires occupés de l'Est. Le train suivait un itinéraire à peine dévié de celui qu'empruntait l'Orient Express quand ma famille rendait régulièrement visite à la branche parisienne (et française de nationalité). Finalement, le destin de ma famille a été scellé à la rampe d'Auschwitz, et je n'ai rien à en dire. Mais sa manie francophile participait d'un rêve commun à tous les Européens du début du Xx ${ }^{\mathrm{e}}$ siècle. La supériorité de la civilisation européenne viendrait de ses compétences langagières déconnectées du territoire. L'utopie de la langue remplacerait le parcours matériel des Lumières. J'émets l'hypothèse que c'est l'échec de la construction du mythe indianiste européen et non pas son succès qui explique le dévoiement idéologique des relations entre langue et nation.

Après la Première Guerre mondiale, le principe des nationalités s'est incarné dans les formes de l'ethnopolitique qui firent disparaître jusqu'au souvenir de cette ambition commune ${ }^{12}$. L'idée que l'exercice de la démocratie devait être

11. «Goethe est et reste un cosmopolite impénitent », mais n'est-ce pas parce qu'il a vécu longtemps en dehors de la politique ?, voir Edmond VERMEIL, « Pourquoi une religion nationale en Allemagne? », Revue de métaphysique et de morale, 46 année, 1, janv. 1939, p. $65-88$, en part. p. 74 .

12. Voir la livraison de la revue The European Legacy, vol. VI, 6, déc. 2001, « Telling political stories. Imaginative constructions of twentieth-century politics », en part. la présentation de Dana ARIELI-Horowitz qui a dirigé ce numéro et son article « The politics of culture in Nazi Germany. Between degeneration and volkism », p. 751-762. 
organisé sur un socle national, même à l'échelle d'un canton, est devenue l'unique terme de l'alternative au communisme. On mit à la trappe les espoirs - peut-être dérisoires - de double appartenance, nationale et européenne, émis par quelques internationalistes. Par un mécanisme redoutable d'un «impérialisme rétroactif ${ }^{13} \gg-$ alors même qu'elles se révélaient incapables de rétablir une pacification durable et une hégémonie diplomatique efficace -, les grandes puissances ont signé le pacte avec le diable.

Celan versus Céline ; il faudra bien que les historiens aussi assument l'intégralité de l'héritage de ce dernier siècle achevé et entreprennent son récit comme le fait Mark Mazower ${ }^{14}$.

Une focalisation sur les points de vue nationaux amène à suivre les mouvements de départ et d'arrivée des immigrants comme un phénomène, certes massif mais déconnecté de l'idée d'une conception commune de la mobilité propre à la civilisation européenne. Il nous est apparu qu'il pourrait exister un caractère commun à l'entreprise de déplacement. Ce ne serait pas la capacité à engager le départ - elle est sans doute propre à tous les hommes qui ont pu rêver d'un avenir meilleur -, mais la capacité à reprendre le cheminement. Ce serait la preuve par la réactivation de la circulation et les « réagencements » identitaires qu'induit ou que complète cette remise en route. Pour qu'il y ait possibilité de réactivation, souvent à des siècles de distance, il faut bien que la disposition de remise en route soit inscrite en divers lieux et en des temps variables. Quand Philippe Joutard propose ici de reprendre sur la longue durée l'examen des rapports entre circulation et identité dans la diaspora huguenote, il montre bien que l'identification collective met en œuvre un principe de circulation des origines, et non une sacralisation de la terre de départ. Caroline Legrand peut alors exposer comment l'Irlande organise une véritable industrie du tourisme mémoriel pour les catholiques et les protestants d'outre-Atlantique. Les jeunes Portugais, suivis par Irène Dos Santos, cherchent à concilier de manière heureuse la patrie familiale et la mobilité par le détour de l'Europe. Et, en Russie, même les cimetières engloutis peuvent revenir en surface, ramenant les vivants vers les lieux de leur expulsion forcée, nous dit Élisabeth GessatAnstett.

13. L'expression est de Rémi BRAGUe, Europe. La voie romaine, Paris, Gallimard, 1999, p. 190. Elle est employée par l'auteur pour qualifier des tentations actuelles de concevoir l'identité européenne. 1999.

14. Mark MAZOwer, Dark Continent. Europe's twentieth century, New York, A. A. Knopf, 
Ce dossier est issu d'une réflexion collective menée, depuis 1999, dans le séminaire de l'École des hautes études en sciences sociales sur l'histoire des minorités migrantes en Europe ${ }^{15}$. Nous avons cherché à repérer dans la «grammaire cachée de l'Europe », les signes d'intelligibilité de certaines mutations actuelles. Des intérêts divers pour la culture de la mobilité, conçue comme une forme sociale relevant d'une histoire totale, nous ont amenés à formuler l'hypothèse du principe de circulation européen, complémentaire de la fabrique des identités nationales ${ }^{16}$.

Car, finalement, en période de paix relative, toutes sortes de formules souples favorisant des dynamiques de recomposition familiale et personnelle étaient en usage. La propension à rêver du voyage n'est donc pas la marque existentielle de minorités migrantes labellisées. Mais l'effort remarquable entrepris par les hommes du XVIII ${ }^{\mathrm{e}}$ siècle pour concevoir une civilisation européenne admettant la tension entre cosmopolitisme et enracinement n'a guère eu de postérité déclarée. Cela ne veut pas dire que le cosmopolitisme européen, conçu comme une dialectique de l'individu agissant entre le local et l'universel par le détour d'une civilisation commune, ne soit réductible aux dénonciations des tristes épigones « fin de siècle » du complot universel contre les patries ${ }^{17}$.

Henriette AssÉO (janvier 2002).

15. Nous voudrions remercier pour leur aide : Dominique Lassaigne, Jean-Louis Georget, Christian Josckhe, Sophie Fesdjian, Dzovinar Kévonian, Caroline Douki, Jean-Pierre Vittu et Joanne Vajda, et tous les participants du séminaire qui ont contribué à cette réflexion.

16. Anne-Marie ThIESSE, La Création des identités nationales, Europe XVIII ${ }^{e}-X X^{e}$ siècles, Paris, Seuil (L’Univers historique), 1999.

17. Steven Vertovec et Robin Cohen, éd., Conceiving cosmopolitanism, Oxford, Oxford University Press, 2002. 